\title{
The androgen receptor and stem cell pathways in prostate and bladder cancers (Review)
}

\author{
KATARZYNA MARCINKIEWICZ ${ }^{1}$, KYMORA B. SCOTLAND ${ }^{1}$, STEPHEN A. BOORJIAN ${ }^{2}$, EMELI M. NILSSON ${ }^{3,4}$, \\ JENNY LIAO PERSSON ${ }^{3}$, PER ANDERS ABRAHAMSSON ${ }^{5}$, CINZIA ALLEGRUCCI $^{4}$, IEUAN A. HUGHES ${ }^{6}$, \\ LORRAINE J. GUDAS ${ }^{1}$ and NIGEL P. MONGAN ${ }^{1,4}$ \\ ${ }^{1}$ Department of Pharmacology, Weill Cornell Medical College, New York, NY 10065; ${ }^{2}$ Department of Urology, \\ Mayo Clinic, Rochester MN, USA; ${ }^{3}$ Division of Experimental Cancer Research, Department of Laboratory Medicine, \\ Clinical Research Center, Lund University, SE-20502 Malmö, Sweden; ${ }^{4}$ Faculty of Medicine and Health Sciences, \\ School of Veterinary Medicine and Science, University of Nottingham, Sutton Bonington Campus, LE12 5RD, UK; \\ ${ }^{5}$ Department of Urology, Lund University, Skåne University Hospital, SE-20502 Malmö, Sweden; ${ }^{6}$ University \\ Department of Paediatrics, Addenbrookes Hospital, University of Cambridge, Hills Rd., Cambridge, UK
}

Received July 1, 2011; Accepted August 12, 2011

DOI: 10.3892/ijo.2011.1212

\begin{abstract}
Bladder cancer is three times more common in men than in women. However, the physiological basis of the male predominance of bladder cancer remains poorly understood. A higher than expected association of prostate and bladder cancers has also been reported which may indicate a common mechanism of carcinogenesis. Consistent with this, androgens and the androgen receptor (AR) play essential roles in prostate carcinogenesis and are believed to play a role in bladder carcinogenesis. There is also evidence implicating cancer stem cells in prostate and bladder cancers. Indeed putative prostate and bladder cancer stem cells share some common molecular features. We highlight key proteins (CD49f, CD133, PTEN, CD44) which are implicated in both prostate and bladder cancers and are enriched in putative prostate and bladder cancer stem cells. We examine published chromatin immuno-precipitation studies analyzing the genome-wide distribution of the AR to identify AR association with, and by inference potential AR-regulation of, these loci. We discuss recent evidence indicating a role for the AR in the splicing of the key urological stem cell protein CD44. We propose a model whereby aberrant $A R$ regulation of these putative stem cell proteins contributes to malignant transformation of prostate and bladder cells. For these reasons we propose that the relationship between androgens and cancer stem cell associated proteins warrants further investigation.
\end{abstract}

Correspondence to: Dr Nigel Mongan, SVMS, University of Nottingham, LE12 5RD, UK

E-mail: nigel.mongan@nottingham.ac.uk

Key words: androgen receptor, cancer stem cell, chromatin immunoprecipitation, CD44, CD133/prominin1, CD49f/ITGA6, PTEN, prostate, bladder

\section{Contents}

1. Introduction

2. Prostate stem cells and cancer

3. Bladder urothelial stem cells and cancer

4. Androgens in prostate and bladder cancer

5. Stem cell related proteins are common to prostate and bladder cancer

6. Androgen receptor occupancy of putative stem cell loci and regulation of $\mathrm{CD} 44$ splicing

7. Future perspectives and conclusions

\section{Introduction}

There is evidence that many adult tissues harbour a limited subset of highly specialized cells which possess the capacity to renew and regenerate adult tissues. These adult stem cells share some of the functional and molecular characteristics of embryonic (ES) and induced pluripotent stem (iPS) cells, including an ability to self renew and differentiate; (1) and references therein. Adult stem cells have now been identified in many tissues, including the prostate gland (2) and urinary bladder $(3,4)$. Some common solid tumors, including of the prostate (5) and bladder (6), are also believed to harbor cancer stem cells (CSCs) which are alternatively referred to as tumor initiating cells (TICs). These CSCs adopt the functional capacity to self renew and differentiate into the tumor's heterogeneous cell types, but lack the growth regulatory pathways of non-malignant stem cells (1). The evidence in support of the CSC hypothesis has been reviewed in detail recently (7).

Bladder and prostate cancers were estimated to account for over 70,000 and 217,000 new cases respectively in the United States in 2010 and thus represent a major clinical challenge (8). Invasive bladder cancers generally portend a guarded prognosis, while metastatic disease remains incurable (9). Similarly no curative therapy exists for metastatic prostate cancer. 
Known risk factors for bladder cancer include advanced age, cigarette smoking, occupational carcinogen exposures, prior radiotherapy and male gender $(8,10)$. In fact, bladder cancer is approximately three times more common in men than in women (8). Moreover, when other established risk factors are controlled for, the male gender-related risk of bladder cancer persists (10). This suggests an androgenic contribution to bladder cancer development, which we and others have investigated (11-14).

A higher than expected clinical association of prostate and bladder cancers has also been reported (15). Specifically, $25-70 \%$ of pathological specimens from patients undergoing radical cystectomy for bladder cancer have been found to contain prostate cancer $(16,17)$. While an increased risk of bladder cancer in patients undergoing radiation therapy for prostate cancer has been described (18), the link between these malignancies has also been shown to be independent of treatment (15). This has been postulated to result from a common carcinogenic pathway, potentially initiated by urinary stasis and chronic inflammation; (15) and references therein. These findings raise the intriguing possibility that at least some prostate and bladder cancers share similar mechanisms of carcinogenesis.

Although there have been important advances in the diagnosis and treatment of these cancers, the underlying molecular events resulting in prostate and bladder cancer initiation remain poorly understood. The bladder and the prostate gland of men share the common embryological origin in the urogenital sinus (19), where androgens have been shown to play important roles during the developmental process (20). Both the prostate (21) and bladder (3) are believed to harbor rare adult stem cell populations which are defined by their functional ability to regenerate the prostate and bladder. Some controversy exists as to the exact molecular identity of prostate and bladder stem cells and whether a stem cell phenotype can be related to the expression of specific protein markers. However, as we will outline below, understanding of urological stem cell function has been advanced by the combined use of expression of specific cell markers coupled with functional characterization of the ability of such cells to differentiate into distinct cell lineages and/or regenerate the prostate or bladder. In this review, we identify common molecular features of putative bladder and prostate stem cells and relate this to new data concerning the potential androgen regulation of these proteins in prostate and bladder cells. These characteristics should be considered in the context of the development of novel therapeutic interventions to prevent, reverse or delay malignant transformation of normal urothelial stem cells; and indeed how the unique molecular characteristics of transformed CSCs may be exploited as novel biomarkers of premalignant lesions.

\section{Prostate stem cells and cancer}

The prostate epithelium is composed of three differentiated cell types (Fig. 1A), luminal epithelial, basal and neuroendocrine cells (reviewed in ref. 22). It has long been known that the prostate gland involutes following androgen withdrawal, but retains the functional capacity to regenerate distinct prostate cell lineages following androgen restoration (23). This regenerative capacity is attributed to an androgen responsive cell which does not require androgens for self renewal. Such androgen-independent cells could persist during multiple rounds of androgen ablation and restoration. Although the exact identity and defining molecular characteristics of the stem cells mobilized to regenerate the prostate are still being refined, the past decade has seen significant advances in our understanding of the roles of these cells in the normal prostate and of how malignant prostate stem cells contribute to localized and metastatic disease. Much of this work has involved using cell surface markers to identify and enable the functional validation of putative stem cells isolated from the mouse prostate. Early studies identified subsets of prostate cells with stem celllike characteristics, based on the expression of $\alpha 2 \beta 1$ integrin (21) and CD133 (24). These methodologies were also used to enrich a stem cell antigen-1-positive $\left(\mathrm{Scal}^{+}\right)$murine prostate cell population which could regenerate the prostate (25). These cells have been further characterized by the absence of the CD45, CD31 and Ter119 lineage markers and the presence of CD49f/ $\alpha 6$ integrin/ITGA6 (2) and Trop2 (26). Subsequent studies utilized the expression of basal cell surface markers to enable identification and isolation of putative prostate stem cells $(2,21,24-26)$. These cells have been shown to possess stem cell-like functional characteristics, including androgen independence, self renewal and multi-lineage differentiation capacity, and are localized to the basal membrane of the mouse prostate gland $(2,21,24,26)$. Leong and colleagues identified a distinct population of mouse prostate cells, also associated with the prostate basal layer; these cells express stem cell factor (cKit/CD177), in addition to CD133 and Scal, and can regenerate the entire prostate (27). Putative prostate stem cells residing within the basal cell layer are also susceptible to malignant transformation (28-30). However, prostate cancer is histologically characterized by a loss of glandular structures and an increase in luminal cell proliferation suggesting a luminal origin. Consistent with this, androgen-independent luminal cells expressing the Nkx3.1 marker have been identified which possess both regenerative capacity and susceptibility to transformation in the absence of PTEN (31). Therefore, rare cells associated with both the prostate luminal and basal layers have been shown to possess stem cell-like functions and are implicated in prostate carcinogenesis.

\section{Bladder urothelial stem cells and cancer}

The bladder urothelium is composed of three cell layers (Fig. 1B); the umbrella cell layer exposed to the bladder lumen, the intermediate cell layer, and the basal cell layer (reviewed in ref. 32). Functional evidence for the existence of urothelial stem cells includes the ability of the bladder to repair and regenerate following injury $(3,33)$. However, identification of the location within the bladder where these cells reside has proven more difficult to address. Experimental approaches exploiting the distinctive functional characteristics of stem cells, such as slow cycling time and long cellular life, have been applied to enrich putative stem cells from within the bladder cell population. This method enabled the enrichment of a rare population of basal bladder cells which had the enhanced clonogenic capacity typically associated with stem cells (4). These label retaining cells (LRCs) persisted up to one year following labeling indicating a remarkable life span of 
A.

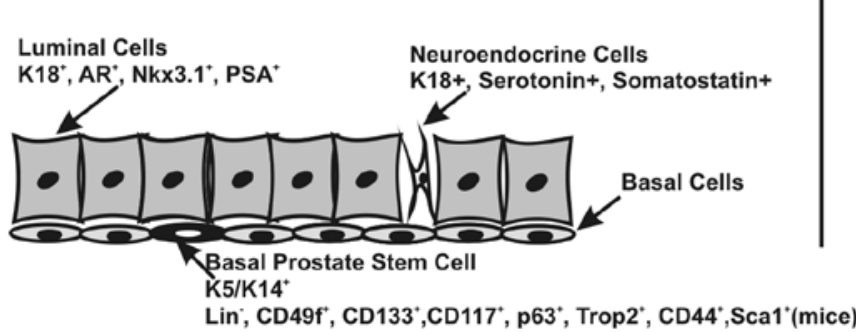

B.

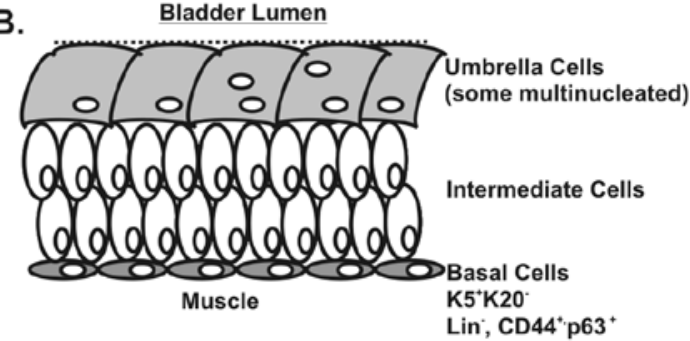

Figure 1. Molecular characteristics of prostate and bladder cell layers. Cellular organization and molecular markers of the prostate (A) and bladder tissue layers (B). Expression of specific cell surface markers has been used to enrich for specific cell types as indicated. Cells associated with both the basal and luminal cell layers are implicated in prostate cancer stem cell function. Bladder cancer tumor initiating cells have been identified associated with the bladder basal cell layer.

these rare cells within the bladder basal layer. Further characterization of this bladder LRC population indicated that these cells were enriched in other markers consistent with a stem cell phenotype, including $\beta 4$-integrin (4).

\section{Androgens in prostate and bladder cancer}

Androgens and the androgen receptor (AR) play complex roles in the earliest stages of male and female urogenital development $(34,35)$. The transcriptional actions of the physiological androgens, testosterone and dihydrotestosterone, are mediated by the AR, a member of the ligand-dependent transcription factor family of nuclear receptors (20). In addition to its role as a regulator of transcriptional activation, the AR has been shown to influence transcript splicing, including splicing of $C D 44$ (36-38). Androgens and the AR play a crucial though complex role in both prostate $(39)$ and bladder $(11,12,14)$ cancers. For example, the AR regulates the activation of distinct transcriptional networks in hormone-dependent and castration-resistant prostate cancer (39). In bladder cancer, both androgens and the AR have been shown to play a role in carcinogenesis in a model system (12) and may represent a potential therapeutic target (40). Although a recent report by Mir and colleagues did not find an association between stage and loss of AR expression in bladder cancer (13), we and others have shown high levels of AR expression in non-invasive tumors, and a progressive loss of expression with increasing pathologic stage has been reported $(11,14)$. Thus, the AR appears to play important roles in the early stages of both prostate and bladder cancers.

\section{Stem cell related proteins are common to prostate and bladder cancer}

Multiple strands of clinical and molecular evidence have implicated the CD49f $(2,41,42)$, CD133 (5,24,43), CD44 $(5,6,44,45)$ and PTEN $(46-51)$ proteins in prostate and bladder cancers. We will discuss the functional and potential clinical significance of each of these proteins in prostate and bladder cancers.

CD49f/ITGA6. CD49f, also designated as integrin- $\alpha 6$ (ITGA6) adhesion molecule, is a cell surface marker that is expressed in stem and progenitor cells from various tissues types including bone marrow, brain, embryo and mammary gland $(52,53)$. The combined use of expression of CD49f/ITGA6 and other stem cell markers such as murine Sca-1 has been applied for purification of prostate stem cells $(2,41)$. This results in an enrichment of prostate stem cell populations with self renewal activity and the ability to form spheres in vitro $(2,41)$. Thus, undifferentiated murine prostate epithelial cells express stem cell markers including Sca1 and CD49f/ITGA6, as well as basal cell markers, including p63 and cytokeratins-5 and -14 , and luminal cell markers, cytokeratins 8 and 18. Similar studies in breast cancer revealed that the stem cell-like subpopulation that expressed CD49f/ITGA6 within the human MCF7 breast cancer cell line had increased tumorigenicity in vitro, compared with those that did not express CD49f/ITGA6 (54). Knockdown of CD49f/ITGA6 causes mammospherederived cells to lose their ability to grow as mammospheres and abrogates their tumorigenicity in xenograft studies (55). These findings suggest that CD49f/ITGA6 is required for the growth and survival of the stem cells, and it is therefore likely to play a key role in oncogenesis. Consistent with this CD49f/ITGA6 has been reported to be overexpressed in bladder cancer and is associated with poorer survival (42).

CD133. CD133 (AC133 or prominin 1) is a plasma membrane protein expressed in embryonic epithelial structures and also found in various adult epithelial cells and hematopoietic/ progenitor cells. Although little is known about the function of CD133 protein, its localisation suggests that it may be involved in the organisation of membrane protrusions and membrane topology. Transcription of CD133 is controlled by five alternative promoters and spliced CD133 variants with different cytoplasmic domains exist in specific tissues (reviewed in ref. 56). Initially identified as a marker of hematopoietic and neural stem cells, CD133 is considered to be a stem cell marker in several other tissues, and it is believed to play a role in the interaction of stem cells to their niche (57). However, the utility of CD133 as a stem cell marker has been questioned by recent evidence indicating CD133 expression is not restricted to stem cells in some tissues (58). However, in normal human prostate $\mathrm{CD}_{133}{ }^{+}$cells represent $\sim 1 \%$ of the entire cell population. These cells are located in the basal layer of the prostate epithelium and colocalize with the same rare cell population expressing high levels of $\alpha 2 \beta 1$. Importantly, CD133 $/ \alpha 2 \beta 1^{\text {high }}$ prostate 
stem cells are capable of reconstituting differentiated prostate acini when injected into nude nice and displayed the greatest proliferative capacity when compared to $\mathrm{CD} 133$ prostate cell populations (24). Molecular studies of isolated $\mathrm{CD} 33^{+} /$ $\alpha 2 \beta 1^{\text {high }}$ cells have identified an expression profile associated with embryonic development, where genes of the Wnt, BMP, TGF $\beta$, SHH and Notch pathways regulate proliferation and differentiation of these stem cells (59).

Prostate CSCs can also be identified as a $\mathrm{CD} 133^{+}$population and retain many features of the normal stem cell counterparts. Combined with the expression of CD44, prostate CSCs (CD44 ${ }^{+} /$ $\left.\alpha 2 \beta 1^{\text {high }} / \mathrm{CD} 133^{+}\right)$, progenitor cells (CD $44^{+} / \alpha 2 \beta 1^{\text {high }} / \mathrm{CD} 133^{-}$) and committed basal cells $\left(\mathrm{CD} 44^{+} / \alpha 2 \beta 1^{\text {low }}\right)$ can be distinguished. The $\mathrm{CD} 44^{+} / \alpha 2 \beta 1^{\text {high }} / \mathrm{CD} 133^{+} \mathrm{CSCs}$ are rare, representing only $0.1-0.3 \%$ of prostate primary tumours and metastases. Only the $\mathrm{CD}_{133^{+}}$fraction of prostate CSCs are capable of extensive proliferation in vitro and can be induced to differentiate into a secretory luminal phenotype expressing cytokeratin 8 , AR and prostatic acid phosphatase (5).

Less is known about the role of CD133 as specific marker of bladder stem cells. To date, only one study reported the identification of bladder CSCs from transitional cell carcinoma based on expression of CD133. Bentivegna and colleagues (43) have shown that urothelial CSCs that can be cultured as urospheres in serum-free conditions and in the presence of growth factors express high levels of CD133, and low levels of cytokeratins-5 and -8 . That only a small proportion of cells in the urospheres express cytokeratin 5 , which has been previously reported to be a bona fide urothelial CSCs marker (6) and that CD133 expression is retained after urosphere cell differentiation, suggests that CD133 mainly identifies committed bladder progenitor cells. Consistent with this, urosphere-derived cells were unable to form tumours in a xenograft mouse model. Taken together these studies suggest that although the value of CD133 expression as marker of prostatic CSCs is well established, more work is needed to clarify the role of CD133 in bladder CSCs.

CD44. CD44 is a transmembrane glycoprotein that functions in cell adhesion (60) and is present in both putative prostate $(5,44,45)$ and bladder $(6)$ stem cells. The $\mathrm{CD} 44^{+}$population of prostate cancer cells has been shown to consist of tumorinitiating cells $(5,45)$. Expression of CD44 splice variants has recently emerged as an important contributor to prostate cancer progression (reviewed in ref. 61). Several groups have found that CD44 expression correlates with metastasis and poor prognosis $(45,62)$. CD44 has recently been shown to be negatively regulated by miR34a, a microRNA frequently lost or reduced in prostate cancer (63). Consistent with this, overexpression of miR34a and the resulting decrease in CD44 protein has been shown to block prostate cancer metastasis (63).

In the bladder, CD44 is expressed in the basal cells, the region postulated to harbor the bladder stem cells (64). Recent reports indicate that a sub-population of $\mathrm{CD} 44^{+}$cells constitutes bladder tumor initiating cells $(6,65)$. Basal cell marker expression was confirmed by demonstrating the expression of cytokeratin 5 in the $\mathrm{CD} 44^{+}$cell subpopulation (6). Chan and colleagues also suggest that CD44 expression in tumor cell subpopulations correlates with muscle invasion and poor prognosis, even in non-muscle invasive tumor specimens (6). It is interesting to speculate that this potential association of CD44

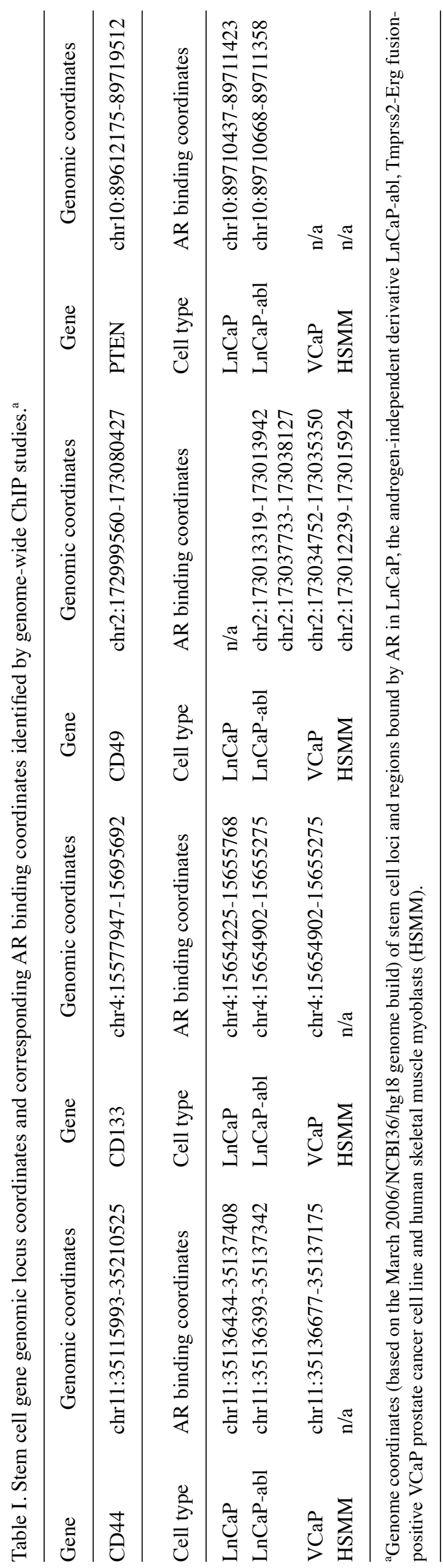




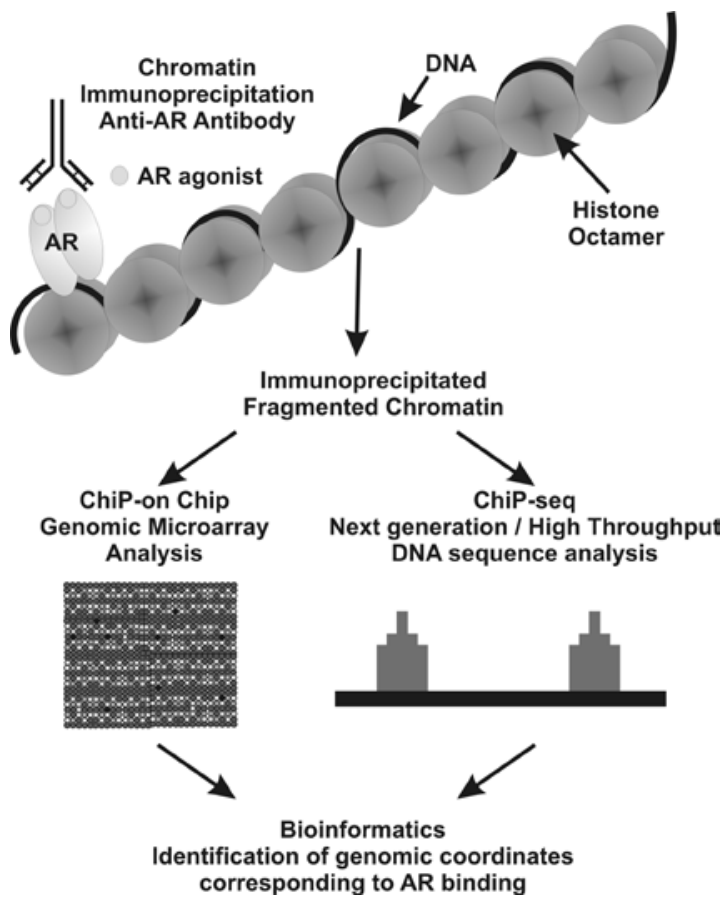

Figure 2. Genomewide chromatin immunoprecipitation (ChIP) assays identify transcription factor binding. Chromatin is composed of DNA and associated histone proteins. The use of antibodies selective to transcription factors such as the AR, permit the isolation of chromatin fragments associated with the AR-complex. The region of DNA bound by the AR complex can be identified either by ChIP coupled with genomic tiling microarray technology (ChIP-onchip) or ChIP coupled with next generation/high throughput DNA sequencing (ChIP-seq).

with poorer outcome may be useful in the future in guiding therapeutic plans for patients with bladder cancer.

Some controversy remains regarding the significance of CD44 expression in aggressive bladder cancers. There is evidence indicating that expression of $C D 44$ is lost or heterogeneous in higher grade bladder tumors $(66,67)$. One possible explanation is that loss of CD44 and the concomitant disruption in cell adhesion may be required for tumor progression. In this way elevated CD44 may have a role in localized cancer and its loss may facilitate invasion. While further research is required to clarify the function of CD44 in bladder tumor progression, current data support the potential of CD44 as a prognostic tool for bladder cancer.

PTEN. Epigenetic silencing of tumor suppressor genes has emerged as an important process in carcinogenesis (68) and occurs in prostate (46-48) and bladder cancers (49-51). One important example in urologic cancer is the PTEN tumor suppressor gene, expression of which is lost in $>50 \%$ of advanced prostate cancers (69,70 and references therein) and some bladder cancers (71,72). PTEN has recently been shown to be essential for the maintenance of hematopoietic stem cell function and prevention of leukaemogenesis (73-75), which suggests that PTEN possesses broader roles in the maintenance of normal stem cell function and inhibition of malignant transformation of adult stem cells. PTEN loss in advanced prostate cancers is associated with progression to androgen-independent disease (70), a phenotypic feature similar to the androgen-independence predicted to be necessary for prostatic stem cell survival
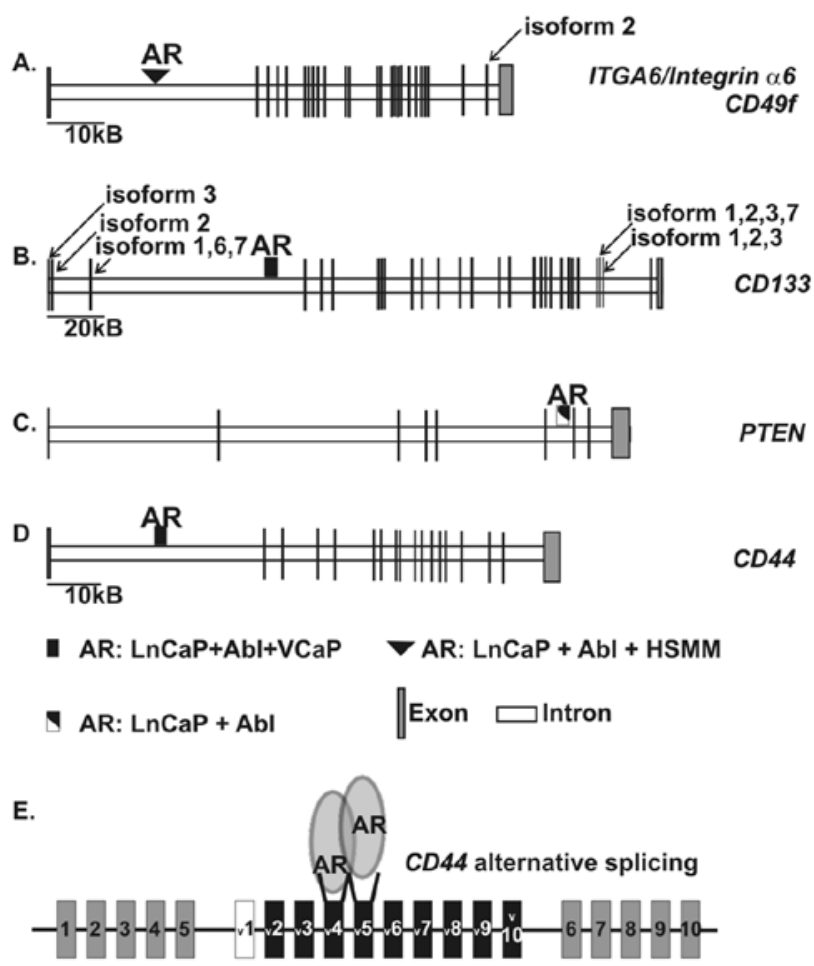

Figure 3. AR binding in stem cell loci and regulation of CD44 splicing. Genome coordinates for AR occupancy in human skeletal muscle myoblasts (accession no.: GSE22076) and LnCaP (accession no.: GSE14028) and VCaP (accession no.: GSE14028) prostate cancer cells were downloaded from the NCBI GEO database. $\mathrm{LnCaP}$ and $\mathrm{LnCaP}$-abl ChIP-chip data were accessed from the Brown lab (http://research4.dfci.harvard.edu/brownlab//datasets/). All genome coordinates were loaded as custom tracks into the UCSC genome browser (http:// genome.ucsc.edu/) and AR occupancy of stem cell loci examined. Using these datasets, we have identified AR occupancy of the CD44 (A), CD49f/integrina6 (B); CD133 (C); and PTEN (D) loci. Exons and introns are indicated Approximate location of $\mathrm{AR}$ binding sites as detected in $\mathrm{LnCaP}$, the androgen independent $\mathrm{LnCaP}$-abl line, $\mathrm{VCaP}$ prostate cancer cells and human skeletal muscle myoblast cells (HSMM) are indicated; based on published findings (39,82-84). (E) AR regulates splicing of the CD44 transcript (36-38). Standard exons (grayscale) and variant exons are indicated in black. Variant exon 1 gives rise to a truncated protein.

as discussed earlier. Consistent with this, deletion of the Pten gene in a mouse model results in expansion of a prostatic stem cell population and tumor initiation (76). Similarly, inactivation of PTEN and p53 cooperatively contributes to muscle invasive bladder cancer in a mouse model (77). In this context it is interesting to note that although expression of PTEN is decreased or altered in only a minority of bladder cancers, loss of the 10q locus and/or PTEN expression has been shown to be associated with a muscle invasive bladder cancer phenotype (78-80) as compared to superficial bladder cancer lesions (81).

\section{Androgen receptor occupancy of putative stem cell loci and regulation of $\mathrm{CD44}$ splicing}

The genomewide distribution of the AR has been determined using chromatin immunoprecipitation (ChIP) studies coupled with genomic microarray analysis (ChIP-chip) $(39,82)$ and ChIP coupled with next generation/high throughput sequencing techniques (ChIP-seq) (Fig. 2; Table I). In this method, antibodies specific to transcription factor of interest, such as the $\mathrm{AR}$, can be used to immunoprecipitate and isolate fragments 
of DNA bound by the AR in vivo. In this way it is possible to identify genomic DNA sequences bound by the AR. ChIP assays permit the unbiased and genomewide determination of transcription factor occupancy when coupled with next generation high throughput DNA sequencing (ChIP-seq), an approach which is now superseding the more established ChIP coupled with genomic tiling microarray (ChIP-chip) method (Fig. 2). These ChIP approaches, when complemented by transcriptomic methods using either mRNA microarray analysis or RNA sequencing, can identify direct transcriptional regulation of target genes. These highly reliable methods have permitted the identification of AR occupancy in a variety of cell types including androgen-responsive $\operatorname{LnCaP}(39,83)$, TMPRSS2-ERG fusion-positive $\mathrm{VCaP}$ prostate cancer cells (83), the androgenindependent derivative of LnCaP, LnCaP-abl (39) and human skeletal muscle myoblasts (HSMM) (82).

ChIP assays have proven to be a powerful technique to determine transcription factor binding and mechanisms of transcriptional regulation $(39,82-84)$. One limitation to these methodologies is that transcription factors can exhibit cell type-specific functions and genomic distributions. However, the consistent identification of AR binding at specific loci in different cell types and across species increases the confidence of functional significance to this AR binding. For example, genomewide ChIP-seq studies have identified over 37,000 loci occupied by agonist bound $\mathrm{AR}$ in $\mathrm{LnCaP}$ and over 12,000 AR-loci in VCaP prostate cancer cells (83). The AR bound loci identified by ChIP-seq (83) in LnCaP included $82 \%$ of the loci previously identified by ChIP-chip in LnCaP (39), demonstrating the reproducibility and robustness of these techniques. Similarly, ChIP-chip analysis identified $>32,000$ DNA regions occupied by the AR in skeletal muscle cells, the majority $(91.53 \%)$ of which are located in intergenic and intronic regions (82). Our analyses of these datasets have identified AR occupancy of the CD44, CD49f/integrin- $\alpha 6, C D 133$ and PTEN loci (Fig. 3A-D). A recent genome-wide analysis of AR in mouse epididymis reveals only a minority of conservation of AR binding sites between human and mouse genomes (84). Interestingly, this study did identify hormone dependent AR occupancy at the mouse CD49f, CD133 and CD44 loci, suggesting potential conservation of AR function at these key loci between these species. Therefore we can infer the potential for AR regulation of these key stem cell loci by revealing here AR occupancy of these genomic regions in a variety of human and mouse cell types. Direct study of androgen regulation of CD49f, CD133, CD44 and PTEN expression and function in prostate and bladder stem cells has yet to be reported. This reflects the technical challenge of isolating and expanding such cells in culture for genome-wide ChIP analyses. However our analysis suggests that further examination of androgen and AR-regulation of stem cell function is needed.

The transcriptional roles of the AR are well understood (14). Less well recognized is the role of AR in regulating alternate splicing of target transcripts (36-38). One important consideration with respect to CD44 and urological cancers is the ability of androgens and AR to regulate alternate splicing of the $C D 44$ transcript (36-38) (Fig. 3E). CSCs appear to differ from normal cells by the expression of distinct CD44 variants (reviewed in ref. 85). Indeed, distinct pro-tumorigenic and metastatic functions have recently been attributed to CD44 variants (86-88), whereas restoration of standard CD44 expression appears to diminish prostate cancer proliferation (89). This raises the interesting possibility that alterations in AR regulation of $C D 44$ splicing may play a role in androgen related cancers, such as prostate and bladder cancer.

\section{Future perspectives and conclusions}

In conclusion, we have identified key proteins implicated in CSCs function which play roles in both prostate and bladder cancers. Furthermore we highlight experimental evidence which indicates the recruitment of the AR to these key loci in prostate cancer cells $(39,83)$ and other cell types $(82,84)$. We discussed how the AR can play a role in the regulation of splicing of a key stem cell protein, CD44, which is implicated in prostate and bladder cancer. In our model, the AR may contribute to the earliest events of prostate and bladder cell transformation and the adoption of a malignant CSC phenotype through the aberrant regulation of $C D 49 f, C D 44, C D 133$ and PTEN. In this way, androgen function may contribute to both prostate carcinogenesis and the gender disparity in bladder cancer incidence. For these reasons we propose that the relationship between androgens and urological stem cell function warrants further investigation.

\section{Acknowledgements}

The authors acknowledge the financial support from the following sources: NIH/NCBI National Research Service Award F31 CA123703-02 (KBS); NIH-F31 award DE21316-01 (KM). IAH is supported by the NIHR Cambridge Biomedical Research Centre. The authors acknowledge the support of the University of Nottingham (NPM, EMN, CA) and a Tegger's Foundation Postdoctoral Fellowship (EMN).

\section{References}

1. Kashyap V, Rezende NC, Scotland KB, et al: Regulation of stem cell pluripotency and differentiation involves a mutual regulatory circuit of the NANOG, OCT4, and SOX2 pluripotency transcription factors with polycomb repressive complexes and stem cell microRNAs. Stem Cells Dev 18: 1093-1108, 2009.

2. Lawson DA, Xin L, Lukacs RU, Cheng D and Witte ON: Isolation and functional characterization of murine prostate stem cells. Proc Natl Acad Sci USA 104: 181-186, 2007.

3. Shin K, Lee J, Guo N, et al: Hedgehog/Wnt feedback supports regenerative proliferation of epithelial stem cells in bladder. Nature 472: 110-114, 2011.

4. Kurzrock EA, Lieu DK, Degraffenried LA, Chan CW and Isseroff RR: Label-retaining cells of the bladder: candidate urothelial stem cells. Am J Physiol Renal Physiol 294: F1415-F1421, 2008.

5. Collins AT, Berry PA, Hyde C, Stower MJ and Maitland NJ: Prospective identification of tumorigenic prostate cancer stem cells. Cancer Res 65: 10946-10951, 2005.

6. Chan KS, Espinosa I, Chao M, et al: Identification, molecular characterization, clinical prognosis, and therapeutic targeting of human bladder tumor-initiating cells. Proc Natl Acad Sci USA 106: 14016-14021, 2009.

7. Clevers H: The cancer stem cell: premises, promises and challenges. Nat Med 17: 313-319, 2011.

8. Jemal A, Siegel R, Xu J and Ward E: Cancer Statistics, 2010. CA Cancer J Clin 60: 277-300, 2010.

9. Messing EM: Urothelial tumors of the bladder. In: CampbellWalsh Urology. Wein AJ (ed). Saunders-Elsevier, Philadephia, pp2407-2446, 2007.

10. Hartge P, Harvey EB, Linehan WM, et al: Unexplained excess risk of bladder cancer in men. J Natl Cancer Inst 82: 1636-1640, 1990. 
11. Boorjian S, Ugras S, Mongan NP, et al: Androgen receptor expression is inversely correlated with pathologic tumor stage in bladder cancer. Urology 64: 383-388, 2004.

12. Miyamoto H, Yang Z, Chen YT, et al: Promotion of bladder cancer development and progression by androgen receptor signals. J Natl Cancer Inst 99: 558-568, 2007.

13. Mir C, Shariat SF, Van Der Kwast TH, et al: Loss of androgen receptor expression is not associated with pathological stage, grade, gender or outcome in bladder cancer: a large multiinstitutional study. BJU Int 108: 24-30, 2011.

14. Kauffman EC, Robinson BD, Downes M, et al: Role of androgen receptor and associated lysine-demethylase coregulators, LSD1 and JMJD2A, in localized and advance human bladder cancer. Mol Carcinog: doi:10.1002/mc.20758:2011 (E-pub ahead of print).

15. Singh A, Kinoshita Y, Rovito PM Jr, et al: Higher than expected association of clinical prostate and bladder cancers. J Urol 173: $1526-1529,2005$.

16. Montie JE, Wood DP Jr, Pontes JE, Boyett JM and Levin HS Adenocarcinoma of the prostate in cystoprostatectomy specimens removed for bladder cancer. Cancer 63: 381-385, 1989.

17. Abbas F, Hochberg D, Civantos F and Soloway M: Incidental prostatic adenocarcinoma in patients undergoing radical cystoprostatectomy for bladder cancer. Eur Urol 30: 322-326, 1996.

18. Boorjian S, Cowan JE, Konety BR, et al: Bladder cancer incidence and risk factors in men with prostate cancer: results from Cancer of the Prostate Strategic Urologic Research Endeavor. J Urol 177: 883-888, 2007.

19. Moore KL and Persaud TVN: The Developing Human: Clinically Oriented Embryology. Saunders, Philadelphia, 2003.

20. Hughes IA, Lim HN, Martin H, et al: Developmental aspects of androgen action. Mol Cell Endocrinol 185: 33-41, 2001

21. Collins AT, Habib FK, Maitland NJ and Neal DE: Identification and isolation of human prostate epithelial stem cells based on alpha(2)beta(1)-integrin expression. J Cell Sci 114: 3865-3872, 2001.

22. Marker PC, Donjacour AA, Dahiya R and Cunha GR: Hormonal, cellular, and molecular control of prostatic development. Develop Biol 253: 165-174, 2003

23. Isaacs JT: In: Benign Prostate Hyperplasia. Rogers CH (ed). NIH, Bethesda, pp85-94, 1987)

24. Richardson GD, Robson CN, Lang SH, Neal DE, Maitland NJ and Collins AT: CD133, a novel marker for human prostatic epithelial stem cells. J Cell Sci 117: 3539-3545, 2004.

25. Burger PE, Xiong X, Coetzee S, et al: Sca-1 expression identifies stem cells in the proximal region of prostatic ducts with high capacity to reconstitute prostatic tissue. Proc Natl Acad Sci USA 102: 7180-7185, 2005.

26. Goldstein AS, Lawson DA, Cheng D, Sun W, Garraway IP and Witte ON: Trop2 identifies a subpopulation of murine and human prostate basal cells with stem cell characteristics. Proc Natl Acad Sci USA 105: 20882-20887, 2008 .

27. Leong KG, Wang BE, Johnson L and Gao WQ: Generation of a prostate from a single adult stem cell. Nature 456: 804-808, 2008

28. Xin L, Lawson DA and Witte ON: The Sca-1 cell surface marker enriches for a prostate-regenerating cell subpopulation that can initiate prostate tumorigenesis. Proc Natl Acad Sci USA 102: 6942-6947, 2005.

29. Lawson DA, Zong Y, Memarzadeh S, Xin L, Huang J and Witte ON: Basal epithelial stem cells are efficient targets for prostate cancer initiation. Proc Natl Acad Sci USA 107: 2610-2615, 2010.

30. Goldstein AS, Huang J, Guo C, Garraway IP and Witte ON: Identification of a cell of origin for human prostate cancer. Science 329: 568-571, 2010

31. Wang X, Kruithof-de Julio M, Economides KD, et al: A lumina epithelial stem cell that is a cell of origin for prostate cancer. Nature 461: 495-500, 2009.

32. Khandelwal P, Abraham SN and Apodaca G: Cell biology and physiology of the uroepithelium. Am J Physiol Renal Physiol 297: F1477-F1501, 2009.

33. Lavelle J, Meyers S, Ramage R, et al: Bladder permeability barrier: recovery from selective injury of surface epithelial cells Am J Physiol Renal Physiol 283: F242-F53, 2002.

34. Shima H, Tsuji M, Elfman F and Cunha GR: Development of male urogenital epithelia elicited by soluble mesenchymal factors. J Androl 16: 233-241, 1995.

35. Shapiro E, Huang HY and Wu XR: Uroplakin and androgen receptor expression in the human fetal genital tract: insight into the development of the vagina. J Urol 164: 1048-1051, 2000 .
36. Rajan P, Gaughan L, Dalgliesh C, et al: The RNA-binding and adaptor protein Sam68 modulates signal-dependent splicing and transcriptional activity of the androgen receptor. J Pathol 215 : 67-77, 2008

37. Sun J, Blair AL, Aiyar SE and Li R: Cofactor of BRCA1 modulates androgen-dependent transcription and alternative splicing. J Steroid Biochem Mol Biol 107: 131-139, 2007.

38. Clark EL, Coulson A, Dalgliesh C, et al: The RNA helicase p68 is a novel androgen receptor coactivator involved in splicing and is overexpressed in prostate cancer. Cancer Res 68: 7938-7946, 2008

39. Wang Q, Li W, Zhang Y, et al: Androgen receptor regulates a distinct transcription program in androgen-independent prostate cancer. Cell 138: 245-256, 2009.

40. Wu JT, Han BM, Yu SQ, Wang HP and Xia SJ: Androgen receptor is a potential therapeutic target for bladder cancer. Urology 75 820-827, 2010.

41. Mulholland DJ, Xin L, Morim A, Lawson D, Witte O and Wu H Lin-Sca- $1^{+}$CD $49 \mathrm{f}^{\text {high }}$ stem/progenitors are tumor-initiating cells in the Pten-null prostate cancer model. Cancer Res 69: 8555-8562, 2009.

42. Grossman HB, Lee C, Bromberg J and Liebert M: Expression of the alpha6beta4 integrin provides prognostic information in bladder cancer. Oncol Rep 7: 13-16, 2000.

43. Bentivegna A, Conconi D, Panzeri E, et al: Biological heterogeneity of putative bladder cancer stem-like cell populations from human bladder transitional cell carcinoma samples. Cancer Sci 101: 416-424, 2010.

44. Liu AY, True LD, LaTray L, et al: Cell-cell interaction in prostate gene regulation and cytodifferentiation. Proc Natl Acad Sci USA 94: 10705-10710, 1997.

45. Patrawala L, Calhoun T, Schneider-Broussard R, et al: Highly purified $\mathrm{CD} 44^{+}$prostate cancer cells from xenograft human tumors are enriched in tumorigenic and metastatic progenitor cells. Oncogene 25: 1696-1708, 2006.

46. Lodygin D, Epanchintsev A, Menssen A, Diebold J and Hermeking H: Functional epigenomics identifies genes frequently silenced in prostate cancer. Cancer Res 65: 4218-4227, 2005.

47. Jeronimo C, Henrique R, Hoque MO, et al: Quantitative RAR $\beta 2$ hypermethylation: a promising prostate cancer marker. Clin Can Res 10: 4010-4014, 2004.

48. Li LC, Carroll PR and Dahiya R: Epigenetic changes in prostate cancer: implication for diagnosis and treatment. J Natl Cancer Inst 97: 103-115, 2005

49. Christoph F, Weikert S, Kempkensteffen C, et al: Regularly methylated novel pro-apoptotic genes associated with recurrence in transitional cell carcinoma of the bladder. Int J Cancer 119: 1396-1402, 2006

50. Kim WJ and Quan C: Genetic and epigenetic aspects of bladder cancer. J Cell Biochem 95: 24-33, 2005.

51. Zhang Z, Karam J, Frenkel E, Sagalowsky A and Hsieh JT: The application of epigenetic modifiers on the treatment of prostate and bladder cancer. Urol Oncol 24: 152-160, 2006.

52. Ramalho-Santos M, Yoon S, Matsuzaki Y, Mulligan RC and Melton DA: 'Stemness': Transcriptional profiling of embryonic and adult stem cells. Science 298: 597-600, 2002.

53. Stingl J, Eirew P, Ricketson I, et al: Purification and unique properties of mammary epithelial stem cells. Nature 439: 993-997, 2006

54. Welm BE, Tepera SB, Venezia T, Graubert TA, Rosen JM and Goodell MA: SCA-1poscells in the mouse mammary gland represent an enriched progenitor cell population. Dev Biol 245: 42-56, 2002.

55. Cariati M, Naderi A, Brown JP, et al: Alpha-6 integrin is necessary for the tumourigenicity of a stem cell-like subpopulation within the MCF7 breast cancer cell line. Int J Cancer 122: 298-304, 2008

56. Wu Y and Wu PY: CD133 as a marker for cancer stem cells: progresses and concerns. Stem Cells Dev 18: 1127-1134, 2009.

57. Bomken S, Fiser K, Heidenreich $\mathrm{O}$ and Vormoor J: Understanding the cancer stem cell. Br J Cancer 103: 439-445, 2010.

58. Shmelkov SV, Butler JM, Hooper AT, et al: CD133 expression is not restricted to stem cells, and both $\mathrm{CD} 133^{+}$and $\mathrm{CD} 133$ metastatic colon cancer cells initiate tumors. J Clin Invest 118: 2111-2120, 2008

59. Shepherd CJ, Rizzo S, Ledaki I, et al: Expression profiling of $\mathrm{CD}_{133^{+}}$and $\mathrm{CD} 133^{-}$epithelial cells from human prostate. Prostate 68: 1007-1024, 2008.

60. Naor D, Sionov RV and Ish-Shalom D: CD44: structure, function, and association with the malignant process. Adv Cancer Res 71: 241-319, 1997.

61. Iczkowski KA: Cell adhesion molecule CD44: its functional roles in prostate cancer. Am J Transl Res 3: 1-7, 2011. 
62. Hurt EM, Kawasaki BT, Klarmann GJ, Thomas SB and Farrar WL: CD44 ${ }^{+} \mathrm{CD} 24-$ prostate cells are early cancer progenitor/ stem cells that provide a model for patients with poor prognosis. Br J Cancer 98: 756-765, 2008.

63. Liu C, Kelnar K, Liu B, et al: The microRNA miR-34a inhibits prostate cancer stem cells and metastasis by directly repressing CD44. Nat Med 17: 211-215, 2011.

64. McKenney JK, Desai S, Cohen C and Amin MB: Discriminatory immunohistochemical staining of urothelial carcinoma in situ and non-neoplastic urothelium: an analysis of cytokeratin 20 , p53, and CD44 antigens. Am J Surg Pathol 25: 1074-1078, 2001.

65. Yang YM and Chang JW: Bladder cancer initiating cells (BCICs) are among EMACD44v6 ${ }^{+}$subset: novel methods for isolating undetermined cancer stem (initiating) cells. Cancer Invest 26: 725-733, 2008

66. Kuncova J, Urban M and Mandys V: Expression of CD44s and $\mathrm{CD} 44 \mathrm{v} 6$ in transitional cell carcinomas of the urinary bladder: comparison with tumour grade, proliferative activity and p53 immunoreactivity of tumour cells. APMIS 115: 1194-1205, 2007

67. Stavropoulos NE, Filliadis I, Ioachim E, et al: CD44 standard form expression as a predictor of progression in high risk superficial bladder tumors. Int Urol Nephrol 33: 479-483, 2001.

68. Jones PA and Baylin SB: The fundamental role of epigenetic events in cancer. Nat Rev Genet 3: 415-428, 2002.

69. Whang YE, Wu X, Suzuki H, et al: Inactivation of the tumor suppressor PTEN/MMAC1 in advanced human prostate cancer through loss of expression. Proc Natl Acad Sci USA 95: 5246-5250, 1998.

70. Bertram J, Peacock JW, Fazli L, et al: Loss of PTEN is associated with progression to androgen independence. Prostate 66 895-902, 2006

71. Yoo LI, Liu DW, Le Vu S, Bronson RT, Wu H and Yuan J: Pten deficiency activates distinct downstream signaling pathways in a tissue-specific manner. Cancer Res 66: 1929-1939, 2006.

72. Cairns P, Evron E, Okami K, et al: Point mutation and homozygous deletion of PTEN/MMAC1 in primary bladder cancers. Oncogene 16: 3215-3218, 1998.

73. Rossi DJ and Weissman IL: Pten, tumorigenesis, and stem cell self-renewal. Cell 125: 229-231, 2006.

74. Zhang J, Grindley JC, Yin T, et al: PTEN maintains haematopoietic stem cells and acts in lineage choice and leukaemia prevention. Nature 441: 518-522, 2006.

75. Yilmaz OH, Valdez R, Theisen BK, et al: Pten dependence distinguishes haematopoietic stem cells from leukaemia-initiating cells. Nature 441: 475-482, 2006.

76. Wang S, Garcia AJ, Wu M, Lawson DA, Witte $\mathrm{ON}$ and $\mathrm{Wu} \mathrm{H}$ : Pten deletion leads to the expansion of a prostatic stem/progenitor cell subpopulation and tumor initiation. Proc Natl Acad Sci USA 103: $1480-1485,2006$
77. Puzio-Kuter AM, Castillo-Martin M, Kinkade CW, et al: Inactivation of $\mathrm{p} 53$ and Pten promotes invasive bladder cancer. Genes Dev 23: 675-680, 2009.

78. Cappellen D, Gil Diez de Medina S, Chopin D, Thiery JP and Radvanyi F: Frequent loss of heterozygosity on chromosome 10q in muscle-invasive transitional cell carcinomas of the bladder. Oncogene 14: 3059-3066, 1997.

79. Aveyard JS, Skilleter A, Habuchi T and Knowles MA: Somatic mutation of PTEN in bladder carcinoma. Br J Cancer 80: 904-908, 1999.

80. Wang DS, Rieger-Christ K, Latini JM, et al: Molecular analysis of PTEN and MXI1 in primary bladder carcinoma. Int J Cancer 88: 620-625, 2000

81. Koksal IT, Yasar D, Dirice E, et al: Differential PTEN protein expression profiles in superficial versus invasive bladder cancers. Urol Int 75: 102-106, 2005.

82. Wyce A, Bai Y, Nagpal S and Thompson CC: Research Resource: The androgen receptor modulates expression of genes with critical roles in muscle development and function. Mol Endocrinol 24: 1665-1674, 2010.

83. Yu J, Mani RS, Cao Q, et al: An integrated network of androgen receptor, polycomb, and TMPRSS2-ERG gene fusions in prostate cancer progression. Cancer Cell 17: 443-454, 2010.

84. Hu S, Yao G, Guan X, et al: Research resource: Genome-wide mapping of in vivo androgen receptor binding sites in mouse epididymis. Mol Endocrinol 24: 2392-2405, 2010.

85. Toole BP: Hyaluronan-CD44 interactions in cancer: paradoxes and possibilities. Clin Cancer Res 15: 7462-7468, 2009.

86. Ghatak S, Hascall VC, Markwald RR and Misra S: Stromal hyaluronan interaction with epithelial CD44 variants promotes prostate cancer invasiveness by augmenting expression and function of hepatocyte growth factor and androgen receptor. J Biol Chem 285: 19821-19832, 2010.

87. Aaltomaa S, Lipponen P, Ala-Opas M and Kosma VM: Expression and prognostic value of CD44 standard and variant v3 and v6 isoforms in prostate cancer. Eur Urol 39: 138-144, 2001.

88. Omara-Opyene AL, Qiu J, Shah GV and Iczkowski KA: Prostate cancer invasion is influenced more by expression of a CD44 isoform including variant 9 than by Muc18. Lab Invest 84: 894-907, 2004.

89. Yang K, Tang Y, Habermehl GK and Iczkowski KA: Stable alterations of CD44 isoform expression in prostate cancer cells decrease invasion and growth and alter ligand binding and chemosensitivity. BMC Cancer 10: 16, 2010. 